\title{
Original
}

\section{Hiperhidrosis primaria, tratamiento mediante simpatectomía por videotoracoscopía.}

\author{
Rodrigo Chamorro-Castro, Bayardo Robelo-Pentzke, Elliott Garita-Jiménez, John Miranda-Chavarría,
} José A. Mainieri-Hidalgo, Carlos Salazar-Vargas.

\section{Resumen}

Introducción: La hiperhidrosis es una condición muy frecuente que dificulta la vida de los pacientes, ya que se sienten o son abiertamente rechazados por las otras personas con las que interactúan. La simpatectomía por toracoscopía se ha convertido en un método efectivo, seguro y asequible para el tratamiento de la hiperhidrosis primaria.

Material y métodos: Se reporta aquí la experiencia con esta técnica en 80 pacientes consecutivos, intervenidos durante un período de 24 meses.

Resultados: Los resultados obtenidos son comparables con los reportados por otros grupos, así como el grado de satisfacción con el procedimiento.

Conclusiones: La simpatectomía por toracoscopía es una intervención sencilla y efectiva para tratar la hiperhidrosis. Se debe trabajar en el perfeccionamiento de la técnica para disminuir la incidencia de la sudoración compensatoria, efecto colateral frecuente y molesto de la intervención.

Descriptores: hiperhidrosis, simpatectomía toracoscópica, sudoración compensatoria.

Key words: hyperhidrosis, thoracoscopic sympathectomy, compensatory sweating.

Recibido: 18 de abril de 2006

Aceptado: 18 de julio de 2006

Servicio Cirugía de Tórax y Cardiovascular, Hospital Dr. Rafael Angel Calderón Guardia, Caja Costarricense de Seguro Social

Abreviaturas: SVT, simpatectomía video toracoscópica; $\mathrm{T}$, torácica;

Correspondencia: Servicio Cirugía de Tórax y Cardiovascular, Hospital Dr. Rafael Ángel Calderón Guardia

ISSN 0001-6002/2006/48/4/190-193 Acta Médica Costarricense, @2006 Colegio de Médicos y Cirujanos
La hiperhidrosis primaria es una condición caracterizada por una producción de sudor en cantidad mayor que la necesaria para regular la temperatura corporal; en algunas personas la cantidad es tan excesiva que representa un verdadero inconveniente para que desarrollen una vida social, laboral y sentimental normal ${ }^{1}$. Dado que existe una variación en cuanto a la producción de sudor en los seres humanos, es difícil establecer una definición cuantitativa exacta. La hiperhidrosis afecta principalmente las manos, las plantas de los pies y las axilas, pero lo común es que existan combinaciones de áreas, como palmo-plantar, palmo-axilar o palmoaxilo-plantar, sin embargo, hay personas que pueden tener localizaciones aisladas, como sudoración axilar excesiva o enrojecimiento o sudoración facial/craneal, solamente. El padecimiento empieza en la infancia y durante la pubertad, persistiendo, en la mayoría de los casos, toda la vida, aunque en algunos la intensidad del problema disminuye a partir de la tercera década de la vida ${ }^{2,3}$. Los pacientes sufren desde el punto de vista psicológico, ya que perciben el rechazo de otras personas causado por su contacto manual en el diario interacctuar, aunado a la falta de un tratamiento médico efectivo y al poco conocimiento de la comunidad médica de métodos invasivos modernos efectivos y ya probados ${ }^{4}$. La etiología se relaciona con la hiperactividad de las fibras simpáticas y el aumento de la respuesta periférica sudomotora ${ }^{4}$, de allí que la simpatectomía torácica se haya recomendado como tratamiento de elección desde 1920 por Kotzareff, 
y luego por Leriche en 1934,6. Kux describió la simpatectomía mediante la toracoscopía clásica, según refiere Blanco et $\mathrm{al}^{1}$. En Costa Rica la primera simpatectomía videotoracoscópica (SVT) fue realizada en el Hospital México en 2000; luego allí se hicieron pocas más y otras esporádicamente en clínicas privadas ${ }^{7}$. En el Hospital R.A. Calderón Guardia se ha iniciado un programa formal de tratamiento de la hiperhidrosis mediante simpatectomía por videotoracoscopía (SVT); se presenta aquí el análisis de los resultados de la intervención en este grupo de pacientes.

\section{Material y métodos}

Del 15 de diciembre de 2003 al 31 de enero de 2006 se realizaron 165 simpatectomias en 80 pacientes consecutivos para el tratamiento de hiperhidrosis primaria palmar, plantar, axilar o generalizada. Los pacientes, en su gran mayoría, eran adultos con hiperhidrosis de diferentes áreas anatómicas, de evolución crónica y que habían tratado durante su vida diversos métodos de tratamiento médico. A estos pacientes se les realizó simpatectomía de T2, T2 y T3, ó T3 y T4 con electrocauterio monopolar. A todos los enfermos se les explicó exhaustivamente su condición y el tratamiento propuesto, así como sus riesgos y posibles complicaciones. Todos firmaron el consentimiento informado y los padres o representantes legales firmaron en los casos de pacientes menores de edad. Los pacientes fueron entrevistados telefónicamente 3 meses después de la intervención y se les leyó un cuestionario que debían responder de manera directa.

\section{La técnica quirúrgica.}

Fue descrita con detalle en otra publicación; en breve es la que sigue ${ }^{8}$. El paciente yace en decúbito supino y en las palmas de las manos se le colocan sensores de temperatura para detectar los cambios antes y después de la sección ganglionar. Luego se intuba con un tubo de doble lumen. Se utilizan 1 ó 2 accesos, dependiendo de la disponibilidad de un toracoscopio en Y, si hay, se hace un solo acceso o puerto en la axila, en la línea de vello, por detrás del borde lateral del pectoral, por el cual se introducen tanto la cámara como el electrocauterio. Si no hay toracoscopio en $\mathrm{Y}$ se hacen 2 incisiones, 1 igual a la descrita y otra en la línea medio clavicular por debajo de la mama. Se colapsa el pulmón ipsilateral y se desplaza hacia abajo. En el ápex de la cavidad puede fácilmente verse la cadena simpática y se identifica su nivel contado las cabezas de las costillas. Se cauteriza la pleura sobre la cadena y lo mismo se hace con el o los ganglios correspondientes, según el tipo de hiperhidrosis de cada paciente. Después de la sección se produce un salto de la temperatura palmar de 1,2 ó hasta de $3^{\circ} \mathrm{C}$. Se re-expande el pulmón y el aire de la pleura se drena con un tubo que se retira al final de la intervención, al cerrar la incisión. El paciente es observado por unas horas y es egresado una que vez que se comprueba radiológicamente la expansión pulmonar.

\section{Resultados}

Se operaron 80 pacientes con un rango de edad de 11 a 48 años y un promedio de 25 años. Un 33\% por ciento (27/80) eran hombres y un 66\% (53/80), mujeres (H: M, 1:2). Preoperatoriamente, el 47,5\% (38/80) de los pacientes tenía sudoración palmo-plantar; el 20\% (16/80), palmoaxilo-plantar; el 12,5\% (10/80), sudoración palmo-axilar; el $10 \%$ (8/80), sudoración generalizada; el 3,75\% (3/80), sudoración palmar, 3,75\% (3/80), sudoración facial y 2,5\% $(2 / 80)$ solo axilar. Setenta pacientes se operaron con internamiento corto y 10 ambulatoriamente. En 2 casos se tuvo que realizar una minitoracotomía unilateral para completar la intervención, por mala visibilidad toracoscópica o por adherencias pulmonares a la pleura parietal en el área de descenso de la cadena simpática. En 74 (92.5\%) individuos la sudoración palmar desapareció. En 6 (7.5\%) pacientes hubo persistencia unilateral, 2 personas no desearon reope-rarse, 3 fueron reoperadas una vez y 1 una fue intervenida unilateralmente 2 veces más, hasta que su sintomatología desapareció.

Dos de los primeros 15 pacientes hicieron un síndrome de Horner unilateral postoperatorio, 1 de los cuales desapareció espontáneamente a los 2 y medio meses. Una paciente hizo causalgia en miembro superior izquierdo. Hubo 2 casos de neumotórax bilateral y 4 de neumotórax unilateral que requirieron sello de agua. El 92,5\% (74/80) de los pacientes presentó sudoración compensatoria, siendo de grado menor en el 63.4\% (45/71); en el 33.8\% (24/71), molesta, y deshabilitante en el 2,8\% (2/71) de los pacientes. El porcentaje de satisfacción del procedimiento fue del 88,75\% (71/80). No hubo mortalidad quirúrgica ni morbilidad significativa.

\section{Discusión}

El ser humano puede mantener una temperatura propia independiente de la ambiental, y para ello tiene un metabolismo y mecanismos termorreguladores dentro de los cuales está la sudoración ${ }^{2}$. Normalmente puede excretarse, en personas de alto rendimiento, hasta alrededor de unos $600 \mathrm{ml} / \mathrm{hr}^{2}$, sin embargo, los pacientes con hiperhidrosis producen una cantidad exagerada o desacorde con su grado de actividad, característicamente usan un pañito de manos para estarse secando. La hiperhidrosis puede aparecer desde la infancia y en la mayoría de las veces en la adolescencia, coincidiendo precisamente con períodos vulnerables del desarrollo, lo que convierte esta condición en un severo problema, ya que es frecuente que los pacientes se sientan 
rechazados, sean temerosos de interactuar y ocasionalmente, hasta tengan ideas suicidas. Si bien es cierto existen diferentes modalidades médicas para tratar esta condición, como administración de toxina botulínica, aplicación local de preparados dermatológicos varios, iontoforesis, etc ${ }^{8}$, ninguna tiene un efecto duradero; la simpatectomía torácica sí tiene un efecto permanente y fue recomendada desde el principio del siglo pasado, apareciendo después la ablación por diferentes abordajes ${ }^{5,6}$, sin embargo, se reporta que durante los últimos 25 años se han publicado más de 200 artículos en la bibliografía en inglés sobre SVT, lo que convierte esta modalidad en el método de elección para el tratamiento definitivo de la hiperhidrosis ${ }^{9}$. Las características demográficas del grupo aquí presentado se asemejan a las de otras series publicadas de pacientes con esta condición: es el doble más frecuente en mujeres y la edad promedio es alrededor de 25 años ${ }^{1,4,10}$. La mayoría de los autores, en sus publicaciones, al reportar el área afectada dan más importancia a la hiperhidrosis palmar, pero lo común es que un individuo sufra hiperhidrosis en varios lugares simultáneamente. Algunas de nuestras pacientes, además de hiperhidrosis palmar, se quejaban de no poder usar sandalias por el exceso de sudoración en los pies, o de no poder hacer presentaciones por la rubicundez o sudoración facial extrema. El área afectada es la que indica el nivel de ablación que debe realizarse. Las distribuciones y orden de hiperhidrosis que se observan en este grupo de individuos son: palmo-plantar>palmo-axilo-plantar>palmo-axilar> generalizada $>$ palmar pura $>$ facial pura. El porcentaje de satisfacción de los pacientes tratados en esta serie fue del $88,75 \%(71 / 80)$.

La sudoración compensatoria es la producción excesiva de sudor en otras áreas anatómicas, que ocurre después de la simpatectomía. Este fenómeno ocurre en áreas no desnervadas y a través de ellas se elimina todo el líquido de sudoración que el cuerpo debiera excretar y que ya no puede eliminar a través de los miembros afectados por el efecto terapéutico de la simpatectomía ${ }^{3,9,11}$. La sudoración compensatoria ocurre en un porcentaje considerable de los individuos operados, y su incidencia varía desde un $1 \%$ hasta un $90 \%$; el reporte depende del grado de tolerancia del individuo o del interrogador. El porcentaje encontrado en este grupo de pacientes (92\%), calza con el indicado en la bibliografía ${ }^{1,12}$. Sin embargo, se debe resaltar que solo 2 personas de todo el grupo presentaron sudoración compensatoria deshabilitante y preferirían no haberse operado. A pesar de ello, el paciente debe ser informado claramente acerca del riesgo de sudoración compensatoria y se debe de trabajar en técnicas que la disminuyan, dado que en algunos casos puede ser deshabilitante.

Ciertos grupos quirúrgicos buscan refinamientos técnicos y anatómicos para, de manera más selectiva, desnervar solo el área problemática de cada paciente, pero los resultados aun no están a la vista ${ }^{11,12}$.
En resumen, la SVT es un método sencillo y efectivo para tratar la hiperhidrosis y los cirujanos con entrenamiento en estas técnicas pueden fácilmente ayudar a gran número de individuos.

\section{Agradecimiento}

A los miembros del Servicio de Anestesia del Hospital R. A. Calderón Guardia, particularmente al Dr. Mario Sánchez Suen, por su valiosa ayuda con la intubación selectiva de todos estos pacientes.

\section{Abstract}

Aim: hyperhidrosis is a frequent condition that complicates the life of the patients. They feel or are actually openly rejected by the persons they interact with. Sympathectomy by videothoracoscopy has become the approach of choice for patients with hyperhidrosis.

Material and methods: we herein report our experience with thoracoscopic sympathectomy in 80 consecutive patients, operated upon during 24 months.

Results: our results are comparable with those reported by other international groups as well as the degree of satisfaction expressed by the patients.

Conclusion: videothoracoscopic sympathectomy is an effective and readily available technique to treat hyperhidrosis. More work is needed to diminish the incidence of compensatory sweating, a frequent and annoying side effect of the procedure.

\section{Referencias}

Blanco AI., Ginel A., Sánchez JM., Barroso R., de la Cruz FJ., García FJ. Tratamiento de la hiperhidriosis mediante simpatectomía torácica videotoracoscópica. Piel 2002; 17: 101-103.

2. Grimalt R., Callejas MA. Tratamiento de la hiperhidrosis focal idiopática con toxina botulínica. Piel 2000; 15:344-350

3. $\quad$ Lai YT., Yang LH., Chio CC., Chen HH. Complications in patients with palmar hyperhdrosis treated with transthoracic endoscopic sympathectomy. Neurosurgery 1997; 41: 110-115

4. Buitrago J., Molins L., Vidal G. Hiperhidrosis palmar y axilar: tratamiento mediante simpatectomía videotoracoscópica. Piel 1999; 14: 394-396

Palumbo LT., Lulu DJ. Anterior transthoracic upper dorsal sympathectomy. Arch Surg 1966; 92: 247-257.

6. Kurchin A., Zweig A., Adar R., Mozes M. Upper dorsal sympathectomy for palmar primary hyperhidrosis by the supraclavicular approach. World J Surg 1997; 1: 667-674. 
7. Alvarado EM., Salazar C., Abed M. Simpatectomía transtorácica mediante video-toracoscopía para el tratamiento de la hiperhidrosis palmar: primer caso reportado en Costa Rica. Acta Med Costar 2000; 42: 212.

8. Chamorro R., Garita E., Mainieri JA., Miranda J., Robelo BJ., Salazar C. Tratamiento de la hiperhidrosis mediante simpatectomía toracoscópica. Experiencia en Costa Rica. Rev Med Broncopul 2006; 3: $27-28$.

9. Licht PB., Pilegaard HK. Severity of compensatory sweating after toracoscopic sympathectomy. Ann Thorac Surg 2004; 78: 427-431
10. Edmonson R., Banerjee A., Rennie JA. Endoscopic transthoracic sympathectomy in the treatment of hyperhidrosis. Ann Surg 1992; 215: 7-11

11. Lee DY., Kim DH., Paik HC. Selective division of t3 rami communicantes in the treatment of palmar hyperhidrosis. Ann Thorac Surg 2004; 78: 1052-1055.

12. Licht PB., Jorgensen OD., Ladegaard L.,Pilegaard HK. Thoracoscopic sympathectomy for axillary hyperhidrosis: the influence of T4. Ann Thorac Surg 2005; 80: 455-460 